\title{
Reduced Lagrangians and analytic solutions in Einstein-æether cosmology
}

\author{
M. Roumeliotis ${ }^{1, \mathrm{a}}$, A. Paliathanasis ${ }^{2,3, \mathrm{~b}}$, Petros A. Terzis $^{1, \mathrm{c}}$, T. Christodoulakis ${ }^{1, \mathrm{~d}}$ (D) \\ ${ }^{1}$ Nuclear and Particle Physics Section, Physics Department, University of Athens, 15771 Athens, Greece \\ ${ }^{2}$ Instituto de Ciencias Físicas y Matemáticas, Universidad Austral de Chile, Valdivia, Chile \\ ${ }^{3}$ Institute of Systems Science, Durban University of Technology, POB 1334, Durban 4000, South Africa
}

Received: 26 November 2018 / Accepted: 8 April 2019 / Published online: 19 April 2019

(C) The Author(s) 2019

\begin{abstract}
We present the solution space of the field equations in the Einstein-æther theory for the case of a $F L R W$ and a locally rotationally symmetric (LRS) Bianchi type $I I I$ space-time. We also find that there are portions of the initial parameters space for which no solution is admitted by the reduced equations. The reduced Lagrangians deduced from the full action are, in general, correctly describing the dynamics whenever solutions do exist. Finally in the FLRW case a curvature singularity exists, while in the type III there are choices of the range of parametres for which there is no curvature singularity.
\end{abstract}

\section{Introduction}

The main context in modified theories of gravity is that new geometric invariants are introduced in the Einstein-Hilbert action of general relativity, for a recent review we refer the reader to $[1,2]$. Modified theories of gravity have been a subject of special interest for the scientific community in recent years because they can describe observable [3-8] phenomena with a geometric approach. More specifically, the geometric invariants which are added to the Einstein-Hilbert action, are introducing new components in the gravitational field equations; these change the dynamics of the field equations in such a way so that the evolution of the latter describes correctly the observed phenomena [9].

In this work, we are interested in the Einstein-æther theory [10-14]. In this theory a unitary time-like vector field, called the æther, is introduced in the gravitational action: This amendment amounts in adding to the Einstein-Hilbert

\footnotetext{
a e-mail: microum@phys.uoa.gr

be-mail: anpaliat@phys.uoa.gr

c e-mail: pterzis@phys.uoa.gr

de-mail: tchris@phys.uoa.gr
}

action a kinetic term quadratic in the covariant derivatives of the field as well as a Lagrange multiplier ensuring that the field is uni-modular. This modification spontaneously breaks the Lorentz symmetry [15], by selecting a preferred frame at each point in space-time while preserving local rotational symmetry. The gravitational field equations are of secondorder and correspond to variations of the action with respect to the metric tensor, the æther field and the Lagrange multiplier. There are various applications of the Einstein-æther theory in cosmological studies. It has been used to describe the later acceleration phase of the universe, as an alternative to dark energy models [16], and also for the early inflationary epoch [17]. On the other hand, Einstein-æther can be seen as the classical limit of Hořava gravity $[18,19]$. Some exact solutions as well as a qualitative analysis of this theory - either in cosmological studies on in static spherical symmetric space-times - can be found in [20-31] and references therein.

A main concept in physics is the mathematical description of the nature. What makes the physical science to differ and stand out from the applied mathematics is the requirement of principles. The principle of stationary action is the most common and describes well the physical phenomena from all areas of nature, from Newtonian mechanics, general relativity and quantum mechanics. A prerequisite for this principle of stationary action to be applied is the existence of a Lagrangian function. This function consists of the Ricci-scalar in the case of Einstein's general relativity, and is supplemented by a kinetic term of the æther field and a Lagrange multiplier ensuring the uni-modular condition for the case of the Einstein-æther theory.

A set of (autonomous) differential equations is not uniquely described by only one Lagrange function [32]. Indeed, it is possible to exist more than one Lagrange functions; for instance, any nonlinear function of the velocity $\dot{q}$, i.e. $f(\dot{q})$, describes the free particle in Newtonian mechanics. 
In general relativity the Lagrangian density is a function of the metric tensor and its first and second derivatives (through the Ricci-scalar). This Einstein-Hilbert action principle correctly reproduces the field equations when all metric components are varied independently. However, when particular metrics are considered, usually by imposing some symmetry, the situation is not straightforward: if the symmetry is imposed to the full action and the redundant coordinates are integrated out, the reduced action can, of course, be varied only with respect to the remaining dependent variables. The ensuing equations of motion may or may not be equivalent to the reduced Einstein equations, i.e the equation resulting from the imposition of the symmetry to the full Einstein's field equations. When they are equivalent, the corresponding reduced Lagrangian is called valid. A well known example of a non valid case is the class B family of Bianchi models (see e.g. $[33,34]$ and references therein). In this case one can search for another Lagrangian correctly reproducing the reduced Einstein equations [35]. The importance of the existence of a Lagrangian description of a given set of equations lies in the reach methods of analytical Mechanics that can be applied in order to study the evolution of the field equations and their integrability [36-42]. Moreover, in the minisuper-space approach (where the reduced Lagrangian takes the form of a point Lagrangian), the quantum analogs of the classical integrals of motion can be used as supplementary conditions to the Wheeler-DeWitt equations [43-49].

The class of Bianchi spatially homogeneous cosmologies contains many important cosmological models, including the standard Friedmann-Lemaître-Robertson-Walker (FLRW) spacetime and the well known Mixmaster universe (Bianchi IX) [50]. In Bianchi models the spacetime manifold is foliated along the time axis, with three dimensional homogeneous hypersurfaces $[51,52]$. There are nine different homogeneous hypersurfaces, each invariant under a corresponding three dimensional Killing algebra [51]. The principal advantage of Bianchi cosmological models is that in these models the gravitational field equations reduce to a system of coupled, ordinary differential equations with independent variable any function $f(t)$ parametrizing the "time-direction". Moreover, the Bianchi models are grouped into two classes, $\mathrm{A}$ and $\mathrm{B}$ (according to the trace $c_{\alpha \beta}^{\alpha}$ of the structure constants tensor being zero or non-zero, respectively); while each class is divided into several types. As earler mentioned, a main difference between these two classes is that for the gravitational field equations which belong to the models of class A the reduced Lagrangian is valid [53], while for the models of class B the corresponding reduced Lagrangian is, in general, not valid except for cases of further reduction (LRS spacetimes, see e.g. [34]). However, for the general type $V$ lineelement, although belonging to class B, a valid lagrangian does exist [35].
In the vacuum and for the Einstein-æther theory we study the existence of analytical solutions and description of the spacetimes with a reduced Lagrangian for two spacetimes of special interest, the homogeneous and isotropic space described by the FLRW line element, and the Bianchi III spacetime. Both spacetimes have a valid reduced action in the Einstein-Hilbert case; though the existence of a reduced Lagrangian is not certain in the Einstein-æther cosmology. The plan of the paper is as follows:

The basic properties and definitions of the Einstein-æther theory are given in Sect. 2. We furthermore derive the field equations for the space-times of our consideration. Sects. 3 and 4 include the main material of our analysis: we determine the conditions under which analytic solutions for the field equations in the Einstein-æther theory exist and present the entire solution space. This is done for the FLRW and the locally rotationally symmetric (LRS) Bianch type II I spacetime, respectively. Furthermore, we determine the conditions so that the reduced Lagrangian is valid and, in doing so, we also classify the space-times here found. The discussion of our results and our conclusions are presented in Sect. 5.

\section{Einstein-æther gravity}

Let $u^{a}$ be a unitary time-like vector field which describes the æther, $u^{a} u_{a}=-1$. Thus the Action integral which, uppon variation of the metric components, gives the gravitational field equations for the Einstein-æther gravity, is given by the following expression [10]

$$
\begin{aligned}
S_{A E}= & \int d^{4} x \sqrt{-g} R+\int d^{4} x \sqrt{-g}\left(K^{\alpha \beta \mu v} u_{\mu ; \alpha} u_{v ; \beta}\right. \\
& \left.+\lambda\left(u^{c} u_{c}+1\right)\right)
\end{aligned}
$$

where $K^{\alpha \beta \mu \nu}$ describes the coupling between the æther field and the gravity, defined as

$K^{\alpha \beta \mu \nu} \equiv c_{1} g^{\alpha \beta} g^{\mu \nu}+c_{2} g^{\alpha \mu} g^{\beta \nu}+c_{3} g^{\alpha \nu} g^{\beta \mu}+c_{4} g^{\mu \nu} u^{\alpha} u^{\beta}$.

with $c_{1}, c_{2}, c_{3}$ and $c_{4}$ being dimensionless constants.

The function $\lambda$ in (2.1) is a Lagrange multiplier which ensures the unitarity of the æether vector field. Finally, $R$ is the Ricci scalar of the underlying space-time with metric $g_{a b}$, and describes the Einstein's General Relativity term in the present theory.

The dynamical equations are obtained by demanding stationarity of the Action integral (2.1) under arbitrary variations with respect to the metric, $\frac{\delta S_{A E}}{\delta g^{a b}}=0$, the æther vector field, $\frac{\delta S_{A E}}{\delta u_{a}}=0$, and the Lagrange multiplier $\lambda, \frac{\delta S_{A E}}{\delta \lambda}=0$. The latter equation provides the unitary constraint for the æther vector field. 
More specifically, variation with respect to the metric tensor gives the gravitational field equations

$G_{a b}=T_{a b}^{\mathfrak{x}}$,

where $G_{a b}$ is the Einstein tensor and $T_{a b}^{\mathfrak{x}}$ is the æther energymomentum tensor defined as

$$
\begin{aligned}
T_{a b}^{\mathfrak{x}}= & \frac{1}{2} g_{\mu \nu} K^{\alpha \beta \rho \sigma} u_{\rho ; \alpha} u_{\sigma ; \beta}+\frac{1}{2} g_{\mu \nu} \lambda\left(g^{\alpha \beta} u_{\alpha} u_{\beta}+1\right) \\
& -c_{1} g^{\alpha \beta}\left(u_{\alpha ; \mu} u_{\beta ; \nu}+u_{\mu ; \alpha} u_{v ; \beta}\right) \\
& -c_{2} g^{\alpha \beta}\left(u_{\mu ; \nu} u_{\alpha ; \beta}+u_{\beta ; \alpha} u_{v ; \mu}\right) \\
& -c_{3} g^{\alpha \beta}\left(u_{\alpha ; \mu} u_{v ; \beta}+u_{\mu ; \alpha} u_{\beta ; \nu}\right)-c_{4} u^{\alpha} u^{\beta} u_{\mu ; \alpha} u_{\nu ; \beta} \\
& -c_{4}\left(g^{\lambda \alpha} u_{\mu} u^{\beta} u_{\lambda ; \nu} u_{\alpha ; \beta}+g^{\beta \lambda} u^{\alpha} u_{\nu} u_{\beta ; \alpha} u_{\lambda ; \mu}\right)-\lambda u_{\mu} u_{v} \\
& +\left(\frac{1}{2} K^{\alpha \beta \lambda \kappa} u^{\rho} u_{\kappa ; \beta} g_{\rho \mu} g_{\lambda \nu}+\frac{1}{2} K^{\alpha \beta \lambda \kappa} u^{\rho} u_{\kappa ; \beta} g_{\rho \nu} g_{\lambda \mu}\right)_{; \alpha} \\
& +\left(\frac{1}{2} K^{\alpha \beta \lambda \kappa} u^{\rho} u_{\kappa ; \beta} g_{\rho \mu} g_{\alpha \nu}+\frac{1}{2} K^{\alpha \beta \lambda \kappa} u^{\rho} u_{\kappa ; \beta} g_{\rho \nu} g_{\alpha \mu}\right)_{; \lambda} \\
& -\left(\frac{1}{2} K^{\alpha \beta \lambda \kappa} u^{\rho} u_{\kappa ; \beta} g_{\lambda \mu} g_{\alpha \nu}+\frac{1}{2} K^{\alpha \beta \lambda \kappa} u^{\rho} u_{\kappa ; \beta} g_{\lambda \nu} g_{\alpha \mu}\right)_{; \rho} .
\end{aligned}
$$

Variation with respect to the æther vector field provides the equation of motion which the vector field $u_{a}$ satisfies, that is,

$$
\begin{aligned}
& c_{4} g^{\mu v} u^{\alpha} u_{v ; \beta} u_{\mu ; \alpha} g^{\kappa \beta}-c_{4} g^{\mu \kappa} g^{\alpha \lambda} u_{\lambda ; \beta} u^{\beta} u_{\mu ; \alpha} \\
& \quad-c_{4} g^{\mu \kappa} u^{\alpha} u_{; \beta}^{\beta} u_{\mu ; \alpha}-K^{\alpha \beta \mu \kappa} u_{\mu ; \alpha ; \beta}+\lambda g^{\alpha \kappa} u_{\alpha}=0
\end{aligned}
$$

one component of which can be used to determine the Lagrange multiplier $\lambda$.

Finally, variation with respect to $\lambda$ gives the condition

$u^{a} u_{a}+1=0$.

It is thus clear that Einstein-æther gravity is a second-order theory; in a four dimensional manifold this system comprises fifteen equations. It is important to mention that in our consideration we have not assumed any matter content.

We continue our analysis by selecting the underlying geometry to be that of (a) FLRW space-time and (b) an LRS Bianchi type II I space-time. In both cases the field equations are reduced to ordinary, coupled differential equations with time as the independent (in principle) dynamical variable.

\section{FLRW}

The generic line element of the FLRW space-time is taken to be

$$
\begin{aligned}
d s^{2}= & -M^{2}(t) d t^{2}+a^{2}(t) \\
& \times\left(\frac{1}{1-k r^{2}} d r^{2}+r^{2} d \theta^{2}+r^{2} \sin ^{2} \theta d \phi^{2}\right),
\end{aligned}
$$

where $a(t)$ denotes the scale factor ( describing the radius of the three dimensional volume), $M(t)$ is the so-called lapse function and $k$ characterises the spatial curvature of the three dimensional hyper-surface $(k=0 \Rightarrow$ flat space, $k=1 \Rightarrow$ space of constant positive curvature $k=-1 \Rightarrow$ space of constant negative curvature).

As far as the æther vector field $u_{a}$ is concerned, the imposition on it of the Killing vector fields of the above line element reduces it to the form $u_{a}=T^{\prime}(t) \delta_{a}^{t} \leftrightarrow u^{a}=-\frac{T^{\prime}(t)}{M(t)^{2}} \delta_{t}^{a}$, where the prime denotes derivative with respect to $t$. This shows that, due to he many symmetries of the model, $u_{a}$ is forced to be curl-free and thus only Hypersurface Orthogonal solutions can exst.

The reason for selecting to define the æther vector field with a derivative is that the Eq. (2.3) become of second order in $t$, a useful occurrence for the reduced Lagrange description.

From the constraint equation (2.6) it straightforwardly follows that

$-\left(\frac{T^{\prime}}{M}\right)^{2}+1=0$

which gives $T(t)=\mu_{1}+\mu_{2} \int M(t) d t,\left(\mu_{2}\right)^{2}=1$. The latter expression quantifies the preferred frame of the comoving observer, and in particular the physical time of the theory $T(t)$.

Furthermore, the only non-vanishing component of (2.5) provides the Lagrange multiplier $\lambda$ :

$\lambda(t)=\frac{3}{a^{2} M^{3}}\left[\left(c_{1}+c_{2}+c_{3}\right) M a^{\prime 2}+c_{2} a\left(a^{\prime} M^{\prime}-M a^{\prime \prime}\right)\right]$.

Finally, with the use of (3.2) and (3.3), the gravitational field equations (2.3) become

$$
\begin{aligned}
& -2 k M^{2}+\left(-2+c_{1}+3 c_{2}+c_{3}\right) a^{\prime 2}=0, \\
& 2 k M^{3}+2\left(-2+c_{1}+3 c_{2}+c_{3}\right) a a^{\prime} M^{\prime} \\
& \quad-\left(-2+c_{1}+3 c_{2}+c_{3}\right) M\left(\left(a^{\prime}\right)^{2}+2 a a^{\prime \prime}\right)=0 .
\end{aligned}
$$

We now use the freedom to choose the time coordinate and, without loss of generality, we select for the lapse function $M(t)=a(t)$; this is possible since under an arbitrary change of time $M(t), a(t)$ transform as density and scalar respectively. Therefore the line element becomes

$d s^{2}=a^{2}(t)\left(-d t^{2}+\frac{1}{1-k r^{2}} d r^{2}+r^{2} d \theta^{2}+r^{2} \sin ^{2} \theta d \phi^{2}\right)$, 
and the gravitational field equations reduce to

$$
\begin{aligned}
& -2 k a^{2}+\left(-2+c_{1}+3 c_{2}+c_{3}\right) a^{\prime 2}=0, \\
& \left(-2+c_{1}+3 c_{2}+c_{3}\right)\left(\left(a^{\prime}\right)^{2}-a a^{\prime \prime}\right)=0 .
\end{aligned}
$$

\subsection{Analytic solutions}

We proceed by investigating the existence of solutions for the field equations (3.7), (3.8). The latter implies that our analysis can be classified according to whether the combination of constants $-2+c_{1}+3 c_{2}+c_{3}$ is equal or different from zero.

\subsubsection{Case 1: $-2+c_{1}+3 c_{2}+c_{3}=0$}

In this case (3.8) is identically satisfied, while (3.7) dictates that

$k=0$,

thus the solution is described by the above equation and any $a(t)$; this peculiarity is reflected into the fact that the corresponding reduced Lagrangian is a total derivative (see next section). The metric becomes

$$
\begin{aligned}
& \left\{\left(-a^{2}, 0,0,0\right),\left(0, a^{2}, 0,0\right),\left(0,0, r^{2} a^{2}, 0\right),\right. \\
& \left.\quad\left(0,0,0, r^{2} a^{2} \sin (\theta)^{2}\right)\right\},
\end{aligned}
$$

and the Lagrange multiplier $\lambda$, is calculated to be

$\lambda(t)=-\frac{3\left(\left(-2+c_{2}\right)\left(a^{\prime}\right)^{2}+c_{2} a a^{\prime \prime}\right)}{a^{4}}$.

The Ricci scalar is computed to be $R=\frac{6 a^{\prime \prime}(t)}{a(t)^{3}}$, and since $a(t)$ is not specified there can be choices for which there are and/or there are not curvatcure singularities

\subsubsection{Case 2: $-2+c_{1}+3 c_{2}+c_{3} \neq 0$}

(1) If $\mathrm{k}=0$ then (3.7) implies $a(t)=c a$ which also satisfies (3.8), while the Lagrange multiplier becomes zero $(\lambda=0)$ and the metric reduces to

$$
\begin{aligned}
& \left\{\left\{-c a^{2}, 0,0,0\right\},\left\{0, c a^{2}, 0,0\right\},\left\{0,0, c a^{2} r^{2}, 0\right\},\right. \\
& \left.\quad\left\{0,0,0, c a^{2} r^{2} \sin (\theta)^{2}\right\}\right\},
\end{aligned}
$$

which of course represents the Minkowski space-time in spherical-polar coordinates.

(2) If $\omega \equiv k *\left(-2+c_{1}+3 c_{2}+c_{3}\right)^{-1}>0$ (the opposite sign $\omega<0$ gives an imaginary part to $a(t)$ and is thus not acceptable), there is a solution $a(t)=m_{1} e^{ \pm \sqrt{2} \sqrt{\omega} t}$ It is noteworthy that a linear combination of the above $a(t)$ with different integration constants $m_{1}, m_{2}$ corresponding to plus and minus signs is not a solution.
The Lagrange multiplier $\lambda$, is calculated to be

$\lambda(t)=\frac{6\left(c_{1}+c_{2}+c_{3}\right) \omega}{a(t)^{2}}$.

The metric becomes

$$
\left\{\left\{-a(t)^{2}, 0,0,0\right\},\left\{0, \frac{a(t)^{2}}{1-k r^{2}}, 0,0\right\},\left\{0,0, r^{2} * a(t)^{2}, 0\right\},\right.
$$$$
\left.\left\{0,0,0, a(t)^{2} r^{2} \sin (\theta)^{2}\right\}\right\} \text {. }
$$

The corresponding Ricci scalar is $R=\frac{6(k+2 \omega) e^{-2 \sqrt{2} t \sqrt{\omega}}}{\mathrm{m} 1^{2}}$ which seems to have a curvature singularity at $t \rightarrow-\infty$. In order for the conclusion to be safe, it is better to turn to the cosmological time $\tau$ where $M(\tau)=1$ : This is achieved by the trasformation $t=\frac{\log \left(\frac{\sqrt{2} \tau \sqrt{\omega}}{\mathrm{ml}}\right)}{\sqrt{2} \sqrt{\omega}}$. In this new time the Ricci scalar reads $R=\frac{3(k+2 \omega)}{\tau^{2} \omega}$, exhibiting the singularity at $\tau=0$. Note also that the constant $(k+2 \omega)$ is essential as it can be seen by eliminating $\tau$ between the above Ricci scalar and $Q \equiv g^{\mu \nu} R_{, \mu} R_{, v}=-\frac{36(k+2 \omega)^{2}}{\tau^{6} \omega^{2}}$; thereby obtaining an invariant relation between $(R, Q)$ in which the constant explicitely appears.

There is also a solution if $c_{1}+3 c_{2}+c_{3}=0$ which is the Minkowski space-time

\subsection{Reduced Lagrangian description}

For the space-time with line element (3.1) the Ricci-scalar is calculated as

$R=\frac{6 a^{\prime \prime}(t)}{a(t) M(t)^{2}}-\frac{6 a^{\prime}(t) M^{\prime}(t)}{a(t) M(t)^{3}}+\frac{6 a^{\prime 2}}{a(t)^{2} M(t)^{2}}+\frac{6 k}{a(t)^{2}}$.

If we substitute in the action (2.1) the above expression, $u^{a}=-\frac{T^{\prime}(t)}{M(t)^{2}} \delta_{t}^{a}$ for the vector field, and ignore a total derivative term we derive the reduced Lagrangian density

$$
\begin{aligned}
\mathcal{L}_{F L R W}= & \frac{a}{M^{7}}\left(M^{8}\left(6 k+a^{2} \lambda\right)-6 a M^{5} a^{\prime} M^{\prime}-c_{4} a^{2} M^{\prime 2} T^{\prime 4}\right. \\
& +M^{6}\left(6 a^{\prime 2}+a\left(-a \lambda T^{\prime 2}+6 a^{\prime \prime}\right)\right) \\
& +2 c_{4} a^{2} M M^{\prime} T^{\prime 3} T^{\prime \prime}-2 a M^{3} M^{\prime} T^{\prime}\left(3 c_{2} a^{\prime} T^{\prime}\right. \\
& \left.+\left(c_{1}+c_{2}+c_{3}\right) a T^{\prime \prime}\right) \\
& +a^{2} M^{2} T^{\prime 2}\left(\left(c_{1}+c_{2}+c_{3}\right) M^{\prime 2}-c_{4}\left(T^{\prime \prime}\right)^{2}\right) \\
& +M^{4}\left(3\left(c_{1}+3 c_{2}+c_{3}\right) a^{\prime 2} T^{\prime 2}+6 c_{2} a a^{\prime} T^{\prime} T^{\prime \prime}\right. \\
& \left.\left.+\left(c_{1}+c_{2}+c_{3}\right) a^{2}\left(T^{\prime \prime}\right)^{2}\right)\right)
\end{aligned}
$$

In order to test the validity of the reduced Lagrangian density $\mathcal{L}_{F L R W}$, we must first derive the corresponding EulerLagrange equations. 
If these equations are algebraically solved for the accelerations, then substituted in the reduced equations of the theory (2.3), (2.5), (2.6) and the resulting equations are identities we shall say that $\mathcal{L}_{F L R W}$, is valid since it correctly reproduces the reduced dynamics.

In the first case $-2+c_{1}+3 c_{2}+c_{3}=0$ after the replacements $T(t)=\mu_{1}+\mu_{2} \int M(t) d t,\left(\mu_{2}\right)^{2}=1, M(t)=a(t)$ the Lagrangian density becomes

$\mathcal{L}_{F L R W}=6 k a^{2}+6 a^{\prime 2}+6 a a^{\prime \prime} \equiv 6 k a^{2}+6\left(a a^{\prime}\right)^{\prime}$.

It is thus evident that the Euler-Lagrange equation gives $k a=0$, so the Lagrangian is valid for $k=0$ and then it does not specify a particular $a(t)$, agreeing with the reduced equations.

In the second case $-2+c_{1}+3 c_{2}+c_{3} \neq 0$ the Lagrangian density (3.16) is always valid.

\section{Bianchi III}

We continue our analysis by considering the diagonal LRS Bianchi III spacetime with fundamental line element

$d s^{2}=-M^{2}(t) d t^{2}+a^{2}(t)\left(d x^{2}+e^{-2 x} d y^{2}\right)+b^{2}(t) d z^{2}$,

which admits the following four Killing vector fields:

$\xi_{1}=\frac{\partial}{\partial y}, \quad \xi_{2}=\frac{\partial}{\partial z}, \quad \xi_{3}=\frac{\partial}{\partial x}+y \frac{\partial}{\partial y}$,

$\xi_{4}=2 y \frac{\partial}{\partial x}+\left(y^{2}-e^{2 x}\right) \frac{\partial}{\partial y}$.

If we apply these symmetries to the æther vector field, demand that the corresponding one form be curl-free so that the solutions are Hypersurface Orthogonal, and also utilize (2.6) we arrive at the final form

$u^{a}=\frac{u_{0}(t)}{-M^{2}} \delta_{t}^{a}+\frac{\lambda_{3}}{b(t)^{2}} \delta_{z}^{a}, \quad u_{0}(t)=\frac{M}{b} \sqrt{\lambda_{3}^{2}+b^{2}}$.

As we did in the previous case of the FLRW we choose the time so that $M(t)=a(t)$ which proves equally helpful in the present case as well With this $u^{a}$ and the line element (4.1) the fourth component of the equation (2.5) assumes the form

$$
\frac{\lambda_{3}\left(b(t) \lambda(t)-\frac{\left(c_{1}+c_{3}\right)\left(a^{\prime}(t) b^{\prime}(t)+a(t) b^{\prime \prime}(t)\right)}{a(t)^{3}}\right)}{b(t)^{3}},
$$

implying that we have to separately investigate the cases $\lambda_{3}=$ $0, \lambda_{3} \neq 0$.
The general approach in trying to reveal the solution space is to algebraically solve two of the equations in terms of the accelerations $a^{\prime \prime}(t), b^{\prime \prime}(t)$ and substitute the result into the rest. In doing so some particular branches appear when the denominators of the corresponding expressions vanish. This may happen either for specific value ranges of the constants or for particular relations among $a(t), b(t)$. In what follows we present all the cases that emerge.

\subsection{Case 1: $\lambda_{3}=0, c_{2}=1$}

The sketch of the solution procedure is as follows:

We solve for $\lambda(t)$ the $(0,0)$ component of $(2.3)$ and substitute into the first component of (2.5) which becomes $-\frac{q a^{\prime 2}}{a(t)^{2}}-\frac{q b^{\prime 2}}{2 b(t)^{2}}-1=0$ where we have replaced $c_{3}=$ $-c_{1}+q-1$.

This expression can be satisfied only when $q<0$. In that case the appropriate scale factors are

$a(t)=e^{\frac{\int \cos (f(t)) d t}{\sqrt{-q}}}, \quad b(t)=e^{\frac{\sqrt{2} \int \sin (f(t)) d t}{\sqrt{-q}}}$

and the rest of the equations (2.3) are satisfied if $f(t)$ obeys the first order differential equation

$f^{\prime}(t)=\frac{\sqrt{2} \cos (f(t))-\sin (f(t))}{\sqrt{-q}}$.

The solution reads

$f(t)=-2 \tan ^{-1}\left(\frac{1}{6}\left(3 \sqrt{6} \tanh \left(\frac{\sqrt{3}\left(\sqrt{-q} t-m_{1} q\right)}{2 q}\right)+3 \sqrt{2}\right)\right)$.

In order to investigate the singularity behaviour we calculate the Ricci scalar in terms of $f(t)$ without inserting the particular expresion above given. The result is

$R=\frac{e^{\frac{1}{3}(-2) \sqrt{2} f(t)}(\sqrt{2} \cos (f(t))-\sin (f(t)))^{2 / 3}(2 \sqrt{2} \sin (2 f(t))+\cos (2 f(t))-2 q-7)}{q}$

The terms enclosed in the two parenthesises are obviously finite; thus the only term that can blow up is the exponential. However one can see that the $f(t)$ above given is bounded as $-3<f(t)<1$ for all $t$ and all ranges of the involved parameters $\left(q, m_{1}\right)$. Therefore we conclude that the described geometry is singularity free.

\subsection{Case 2: $\left(\lambda_{3}=0, c_{2} \neq 1\right.$, and $\left.1+c_{1}+c_{3}=0\right)$ or $\left(\lambda_{3}=0, c_{2} \neq 1\right.$, and $\left.-2+c_{1}+3 c_{2}+c_{3}=0\right)$}

If we follow analogous steps as before we find that the $(3,3)$ component of (2.3) assumes the never vanishing form $e^{\frac{\sqrt{2} q t}{(-q)^{3 / 2}}}$ 
in the first case and $4 e^{-\frac{2 i t}{\sqrt{-q}}}$ in the second. Thus there is no solution in these cases.

$$
\begin{aligned}
& \text { 4.3 Case 3: } \lambda_{3}=0, c_{2} \neq 1 \text {, and } \\
& \left(1+c_{1}+c_{3}\right)\left(-2+c_{1}+3 c_{2}+c_{3}\right) \neq 0
\end{aligned}
$$

If we replace $c_{3}, c_{2}$ as

$c_{3}=-c_{1}+\mu-1, \quad c_{2}=\frac{1}{3}\left(-\frac{\mu \sigma^{2}}{2}-\mu+3\right)$,

we can solve the non-zero component of (2.5) in terms of $\lambda(t)$ and replace into (2.3). As we can easily see the $(0,0)$ component of the latter equation admits a scaling symmetry $a \rightarrow \omega_{1} a, b \rightarrow \omega_{2} b$ and does not contain accelerations. Thus, if we make the replacement $a=e^{\int a_{1} d t}, b=e^{\int b_{1} d t}$ the aforementioned equation becomes

$$
\begin{aligned}
& \frac{1}{12}\left(4 \mu\left(\sigma^{2}+2\right) a_{1}(t) b_{1}(t)+4 \mu\left(\sigma^{2}-1\right) a_{1}(t)^{2}\right. \\
& \left.\quad+\mu\left(\sigma^{2}-4\right) b_{1}(t)^{2}-12\right)=0 .
\end{aligned}
$$

which being a quadratic form in $a_{1}(t), b_{1}(t)$ can be solved by

$$
\begin{aligned}
& a_{1}(t)=\frac{2 \cosh (f(t))}{\sqrt{3 \mu} \sigma}-\frac{\sigma(2 \sinh (f(t)))}{2(\sqrt{3 \mu} \sigma)}, \\
& b_{1}(t)=\frac{\sigma(2 \sinh (f(t)))}{\sqrt{3 \mu} \sigma}+\frac{2 \cosh (f(t))}{\sqrt{3 \mu} \sigma}
\end{aligned}
$$

If we substitute the above given values of $a_{1}(t), b_{1}(t)$ into the rest of (2.3) we find that a solution exists if $f(t)$ satisfies the differential equation

$f^{\prime}(t)=-\frac{1}{\sqrt{3 \mu} \sigma}(\sigma \cosh (f(t))+4 \sinh (f(t)))$,

with solution

$$
\begin{aligned}
& f(t)=-2 \tanh ^{-1} \\
& \times\left(\frac{4}{\sigma}-\frac{\sqrt{\sigma-4} \sqrt{\sigma+4} \tan \left(\frac{1}{6}\left(3 c_{1} \sqrt{\sigma-4} \sqrt{\sigma+4}-\frac{\sqrt{3} \sqrt{\sigma-4} \sqrt{\sigma+4} t}{\sqrt{\mu} \sigma}\right)\right)}{\sigma}\right) .
\end{aligned}
$$

Thus the final solution is

$$
\begin{aligned}
& a(t)=e^{-\frac{6 \sigma f(t)}{\sigma^{2}-16}}(\sigma \cosh (f(t))+4 \sinh (f(t)))^{\frac{\sigma^{2}+8}{\sigma^{2}-16}}, \\
& b(t)=e^{\frac{6 \sigma f(t)}{\sigma^{2}-16}}(\sigma \cosh (f(t))+4 \sinh (f(t)))^{-\frac{2\left(\sigma^{2}-4\right)}{\sigma^{2}-16}},
\end{aligned}
$$

where $f(t)$ is to be replaced by the above given value. The singularity behaviour is qualitatively similar to the previous non-trivial case when the ranges of the constants appearing in $f(t)$ are appropriately restricted. Otherwise the geometry developes singularities at finite $t$. These cases exhaust the assumption $\lambda_{3}=0$.

We are thus left to examine the case $\lambda_{3} \neq 0$ The strategy is now to solve the $(0,0)$ component of $(2.3)$ for the Lagrange multiplier $\lambda(t)$ and substitute into the two components of (2.5). In doing so some branches appear:

4.4 Case 4: $\lambda_{3} \neq 0 c_{1}+c_{2}+c_{3}=0 c_{2}=0$

In this case $\lambda(t)$ reads

$\lambda(t)=\frac{b(t)\left(-2 a(t) a^{\prime}(t) b^{\prime}(t)-b(t) a^{\prime 2}+a(t)^{2} b(t)\right)}{a(t)^{4}\left(b(t)^{2}+\lambda_{3}^{2}\right)}$.

When this is substituted into the first and the fourth component of (2.5), we end up with the single equation.

$-\frac{2 a^{\prime}(t) b^{\prime}(t)}{a(t) b(t)}-\frac{a^{\prime 2}}{a(t)^{2}}+1=0$.

As before, there is the scaling symmetry and thus we can make the replacement

$a(t)=e^{\int \frac{\sinh (f(t))}{\sqrt{3}} d t+\int \frac{\cosh (f(t))}{\sqrt{3}} d t}$,

$b(t)=e^{\int \frac{\cosh (f(t))}{\sqrt{3}} d t-2 \int \frac{\sinh (f(t))}{\sqrt{3}} d t}$

which satisfies the equation. As far as the equations (2.5), (2.3) are concerned, a solution will exist after the above replacement, if the unique differential equation

$f^{\prime}(t)=\frac{\cosh (f(t))-2 \sinh (f(t))}{\sqrt{3}}$

is satisfied.

Finally, the solution of (4.17) is determined to be

$f(t)=2 \tanh ^{-1}\left(2-\sqrt{3} \tanh \left(\frac{1}{2}\left(\sqrt{3} m_{1}+t\right)\right)\right)$.

In this case the Ricci scalar is calculated as $R=0$ while the Kretsmann is $K r \equiv R^{a b c d} R_{a b c d}=\frac{1}{12}\left(e^{2 f(t)}-3\right)^{6}$. This indicates the existence of singularity at values of $t$ depending on the constsnt $m_{1}$. 
4.5 Case 5: $\lambda_{3} \neq 0 c_{1}+c_{2}+c_{3}=0 c_{2}=1$

We now solve the forth component of (2.5) for the Lagrange multiplier $\lambda(t)$ obtaining

$\lambda(t)=-\frac{a^{\prime}(t) b^{\prime}(t)+a(t) b^{\prime \prime}(t)}{a(t)^{3} b(t)}$.

If we substitute the above $\lambda(t)$ into the first component of (2.5), we end up with

$a^{\prime \prime}(t)=\frac{a^{\prime}(t) b^{\prime}(t)}{b(t)}+\frac{a^{\prime 2}}{a(t)}$,

If we substitute the above (4.20) into (2.3) we observe that there is no solution since its $(0,0),(3,3)$ components become the impossible equations $-\frac{\lambda 3^{2} a^{\prime 2}}{a(t)^{2} b(t)^{2}}-1=$ $0, \frac{\lambda 3^{2} a^{\prime 2}+a(t)^{2} b(t)^{2}}{a(t)^{4}}=0$ respectively.

4.6 Case 6: $\lambda_{3} \neq 0 c_{1}+c_{2}+c_{3}=0 c_{2} \neq 0, c_{2} \neq 1$

Since $\lambda_{3} \neq 0, c_{2} \neq 0$, we can solve the fourth component of (2.5) for the Lagrange multiplier $\lambda(t)$ resulting in

$\lambda(t)=-\frac{c_{2}\left(a^{\prime}(t) b^{\prime}(t)+a(t) b^{\prime \prime}(t)\right)}{a(t)^{3} b(t)}$.

If we replace (4.21) into the first component of (2.5) and solve it for $a^{\prime \prime}(t)$ we obtain

$a^{\prime \prime}(t)=a^{\prime}(t)\left(\frac{a^{\prime}(t)}{a(t)}+\frac{b^{\prime}(t)}{b(t)}\right)$.

If $a(t)$ is constant there is no solution since the $(0,0)$ component of (2.3) becomes $-1=0$. For $a(t)$ non-constant, after the replacement of (4.22) into (2.3), its $(2,2)$ component gives

$\frac{\left(c_{2}-1\right)\left(a^{\prime}(t) b^{\prime}(t)+a(t) b^{\prime \prime}(t)\right)}{a(t) b(t)}=0$,

which is satisfied by the first integral $a(t) b^{\prime}(t)=m$.

If $m=0$ then $b(t)=\mathrm{cb}$ and the replacement to the (2.3) make the $(0,0)$ component

$\frac{a^{\prime 2}\left(-\left(c_{2}-1\right) c b^{2}-c_{2} \lambda_{3}^{2}\right)-c b^{2} a(t)^{2}}{c b^{2} a(t)^{2}}=0$.

This equation is easily integrated resulting in the overall solution

$a(t)=m_{1} e^{\frac{c b t \epsilon}{\sqrt{-c_{2} c b^{2}-c_{2} \lambda_{3}^{2}+c b^{2}}}}, \quad b(t)=c b$.
The Ricci scalar is $R=-\frac{2 c_{2}\left(\mathrm{cb}^{2}+\lambda_{3}^{2}\right) e^{-\frac{2 \mathrm{cb} t}{\sqrt{-c_{2} \mathrm{cb}^{2}-c_{2} \lambda_{3}^{2}+\mathrm{cb}^{2}}}}}{\mathrm{ml}^{2}\left(\left(c_{2}-1\right) \mathrm{cb}^{2}+c_{2} \lambda_{3}^{2}\right)}$ and is seen to tend to infinity at $t \rightarrow-\infty$.

If $m \neq 0$ then the integral can be written as $b^{\prime}(t)=$ $\frac{m}{a(t)}$ which reduces the first component of (2.5) to

$$
\frac{2 c_{2} \sqrt{b(t)^{2}+\lambda_{3}^{2}}\left(-a(t) b(t) a^{\prime \prime}(t)+b(t) a^{\prime 2}+m a^{\prime}(t)\right)}{a(t)^{5} b(t)^{2}}=0 .
$$

If the coefficient of $b(t) a^{2}-a(t) a^{\prime \prime}(t)$ vanishes then the above equation dictates $a(t)=\mathrm{ca}$ and thus $b(t)=\frac{m t}{c a}+$ $m_{1}$ which makes the $(0,0)$ component of $(2.3)-1=0$ indicating that there is no solution.

Otherwise if $a^{\prime 2}-a(t) a^{\prime \prime}(t) \neq 0$ then (4.26) can be solved for $b(t)$ giving

$b(t)=-\frac{m a^{\prime}(t)}{a^{\prime 2}-a(t) a^{\prime \prime}(t)}$.

We substitute (4.27) into the integral $b^{\prime}(t)=\frac{m}{a(t)}$ and we obtain two valid solutions for $a(t)$.

Finally we use the (4.27) obtaining two different solutions with the final form

$a(t)=\frac{e^{-\sqrt{m_{2}}\left(m_{3}+t\right)}\left(2 m_{1} m_{2}+e^{\sqrt{m_{2}}\left(m_{3}+t\right)}\right)^{2}}{4 m_{2}^{2}}$,

$b(t)=-\frac{m \sqrt{\mathrm{m} 2}\left(2 \mathrm{~m} 1 \mathrm{~m} 2-e^{\sqrt{\mathrm{m} 2}(\mathrm{~m} 3+t)}\right)}{\mathrm{m} 1\left(2 \mathrm{~m} 1 \mathrm{~m} 2+e^{\sqrt{\mathrm{m} 2}(\mathrm{~m} 3+t)}\right)}$

$a(t)=\frac{e^{-\sqrt{m_{2}}\left(m_{3}+t\right)}\left(2 m_{1} m_{2} e^{\sqrt{m_{2}}\left(m_{3}+t\right)}+1\right)^{2}}{4 m_{2}^{2}}$,

$b(t)=\frac{m \sqrt{\mathrm{m} 2}\left(2 \mathrm{~m} 1 \mathrm{~m} 2 e^{\sqrt{\mathrm{m} 2}(\mathrm{~m} 3+t)}-1\right)}{\mathrm{m} 1\left(2 \mathrm{~m} 1 \mathrm{~m} 2 e^{\sqrt{\mathrm{m} 2}(\mathrm{~m} 3+t)}+1\right)}$

where $m_{2}=-\frac{c_{2} \lambda_{3}{ }^{2} m_{1}^{2}+m^{2}}{\left(c_{2}-1\right) m^{2}}$ in both the above solutions

In both these cases the Ricci scalar has the genaral form $R=\frac{A \exp (2 B(\mathrm{~m} 3+t))}{(C \exp (B(\mathrm{~m} 3+t))+D)^{4}}$, where $A, B, C, D$ are functions of the parameters appearing in the solutions. Hence there are ranges of the parameters for which $C, D$ became positive and thus the above scalar is finite.

Finally there is the somewhat curious case in which we solve the integral with respect to $a(t)$ i.e. take $a(t)=\frac{m}{b^{\prime}(t)}$. Then we may solve algebraically the first component of the (2.5) for $b^{(3)}(t)$

$b^{(3)}(t)=\frac{b^{\prime \prime 2}}{b^{\prime}(t)}+\frac{b^{\prime}(t) b^{\prime \prime}(t)}{b(t)}$.

The apparent branch $b(t)=m_{1} t+m_{2}$ leads to $a(t)=c a$ and has been earlier seen to lead to no solution. 
If we substitute $(4.30)$ and $a(t)=\frac{m}{b^{\prime}(t)}$, the $(0,0)$ component of (2.3) gives us

$$
-\frac{c_{2} \lambda_{3}{ }^{2} b^{\prime \prime 2}+b(t)^{2}\left(\left(c_{2}-1\right) b^{\prime 2}+b^{\prime 2}\right)-2\left(c_{2}-1\right) b(t) b^{\prime 2} b^{\prime \prime}(t)}{b(t)^{2} b^{\prime 2}}=0 .
$$

The solution of (4.31) as well as $a(t)=\frac{m}{b^{\prime}(t)}$ gives the final form of the solution

$a(t)=\frac{2\left(c_{2}-1\right)^{2} m m_{1} \cos ^{2}\left(\frac{\left(m_{2}+t\right) \sqrt{c_{2}} \lambda_{3}^{2}+\left(c_{2}-1\right)^{2} m_{1}^{2}}{2\left(c_{2}-1\right)^{3 / 2} m_{1}}\right)}{c_{2} \lambda_{3}{ }^{2}+\left(c_{2}-1\right)^{2} m_{1}{ }^{2}}$.

The general form of the Ricci scalar is $R=\frac{A}{\cos \left(B\left(m_{2}+t\right)\right)^{4}}$ which obviously diverges at finite intervals of $t$.

We continue with the final case.

\subsection{Case 7: $\lambda_{3} \neq 0 c_{1}+c_{2}+c_{3} \neq 0$}

We replace $c_{3}=-c_{1}-c_{2}+q$ where $q \neq 0$. Then the fourth component of (2.5) leads to the following form of the Lagrange multiplier $\lambda(t)$

$\lambda(t)=-\frac{\left(c_{2}-q\right)\left(a^{\prime}(t) b^{\prime}(t)+a(t) b^{\prime \prime}(t)\right)}{a(t)^{3} b(t)}$,

If we substitute (4.33) to the first component of (2.5) and to the $(3,3)$ component of $(2.3)$, a system of equations is created which can always be solved in terms of $a^{\prime \prime}(t), b^{\prime \prime}(t)$.

$$
\begin{aligned}
a^{\prime \prime}(t)= & \frac{1}{4 a(t) b(t)^{2}\left(b(t)^{2}+\lambda_{3}^{2}\right)} \\
& \times\left(4 c_{2} a(t) b(t) a^{\prime}(t)\left(b(t)^{2}+\lambda_{3}^{2}\right) b^{\prime}(t)\right. \\
& +2 a^{\prime 2}\left(b(t)^{2}+\lambda_{3}^{2}\right)\left(b(t)^{2}\left(c_{2}+q+1\right)\right. \\
& \left.+\lambda_{3}^{2}\left(c_{2}+q\right)\right) \\
& \left.+a(t)^{2} b(t)^{2}\left(q b^{\prime 2}+2 b(t)^{2}+2 \lambda_{3}^{2}\right)\right) \\
b^{\prime \prime}(t)= & -\frac{1}{2 q a(t)^{2} b(t)^{3}\left(b(t)^{2}+\lambda_{3}^{2}\right)} \\
& \times\left(2 a ( t ) b ( t ) a ^ { \prime } ( t ) ( b ( t ) ^ { 2 } + \lambda _ { 3 } ^ { 2 } ) b ^ { \prime } ( t ) \left(b(t)^{2}\right.\right. \\
& \left.\times\left(2 c_{2}^{2}-2 c_{2}+q\right)+2\left(c_{2}-1\right) c_{2} \lambda_{3}^{2}\right) \\
& +2 a^{\prime}(t)^{2}\left(b(t)^{2}+\lambda_{3}^{2}\right)^{2}\left(b(t)^{2}\left(c_{2}^{2}+c_{2}(q-1)-2 q\right)\right. \\
& \left.+c_{2} \lambda_{3}^{2}\left(c_{2}+q\right)\right) \\
& +a(t)^{2} b(t)^{2}\left(b(t)^{2}\left(\left(c_{2}-2\right) q b^{\prime}(t)^{2}+4 c_{2} \lambda_{3}^{2}\right)\right. \\
& \left.\left.+c_{2} \lambda_{3}^{2}\left(q b^{\prime}(t)^{2}+2 \lambda_{3}^{2}\right)+2 c_{2} b(t)^{4}\right)\right)
\end{aligned}
$$

By substituting the above values of $a^{\prime \prime}(t), b^{\prime \prime}(t)$ into the (2.3) we find only the two different equations $(0,0)=0$ and $(1,1)=0$ quadratic in $a^{\prime}(t), b^{\prime}(t)$. We can solve the $(0,0)=0$ equation for $b^{\prime 2}$ and substitute the result into the first equation of (4.34), thus obtaining the very simple equation

$\frac{a^{\prime \prime}(t)}{a(t)}-\frac{a^{\prime}(t) b^{\prime}(t)}{a(t) b(t)}-\frac{a^{\prime 2}}{a(t)^{2}}$.

This equation has the scaling symmetry and can thus be reduced to first order by the use of the replacement $a(t)=$ $e^{\int a_{1}(t) d t}$ and ultimately be solved in terms of $a(t)$ with the result $a(t)=e^{m_{1} \int b(t) d t}$

If we substitute this $a(t)$ into $(0,0)$ and $(1,1)$ components of (2.3) and eliminate $b^{2}$ we obtain the following equation

$$
\begin{aligned}
& 2 m_{1}\left(2 c_{2}{ }^{2}-c_{2}(q+4)-q^{2}+q+2\right) b^{\prime}(t) \\
& \quad+m_{1}{ }^{2} b(t)^{2}\left(2 c_{2}{ }^{2}-c_{2}(q+4)-q^{2}+q+2\right) \\
& \quad+2 c_{2}{ }^{2} \lambda_{3}{ }^{2} m_{1}{ }^{2}-2 c_{2} \lambda_{3}{ }^{2} m_{1}{ }^{2}-c_{2} \lambda_{3}{ }^{2} m_{1}{ }^{2} q \\
& \quad+2 c_{2}-\lambda_{3}{ }^{2} m_{1}{ }^{2} q^{2}-q-2=0
\end{aligned}
$$

If $m_{1}=0$ then from its definition $a(t)=\mathrm{ca}$ and the above equation becomes $2 c_{2}-q-2=0$ which is equivalent to $c_{1}-c_{2}+c_{3}=-2$.

The first component of (2.5) gives

$b^{\prime \prime}(t)=\frac{b(t) b^{\prime 2}}{b(t)^{2}+\lambda_{3}^{2}}$

We substitute (4.37), $m_{1}=0, a(t)=$ ca and $c_{1}-c_{2}+c_{3}=$ -2 into (2.3) and we end up with one differential equation

$\left(c_{2}-1\right) b^{\prime 2}+b(t)^{2}+\lambda_{3}^{2}=0$,

which can be readily solved resulting in the final form of the solution

$$
\begin{aligned}
& a(t)=c a, \quad b(t)=-\frac{\lambda_{3} \tan \left(\frac{\sqrt{c_{2}-1} t}{1-c_{2}}-m_{1}\right)}{\sqrt{-\tan ^{2}\left(\frac{\sqrt{c_{2}-1} t}{1-c_{2}}-m_{1}\right)-1}} \\
& a(t)=c a, \quad b(t)=\frac{\lambda_{3} \tan \left(\frac{\sqrt{c_{2}-1} t}{1-c_{2}}-m_{1}\right)}{\sqrt{-\tan ^{2}\left(\frac{\sqrt{c_{2}-1} t}{1-c_{2}}-m_{1}\right)-1}} \\
& a(t)=c a, \quad b(t)=-\frac{\lambda_{3} \tan \left(m_{1}+\frac{\sqrt{c_{2}-1} t}{1-c_{2}}\right)}{\sqrt{-\tan ^{2}\left(m_{1}+\frac{\sqrt{c_{2}-1} t}{1-c_{2}}\right)-1}} \\
& a(t)=c a, \quad b(t)=\frac{\lambda_{3} \tan ^{\left(m_{1}+\frac{\sqrt{c_{2}-1} t}{1-c_{2}}\right)}}{\sqrt{-\tan ^{2}\left(m_{1}+\frac{\sqrt{c_{2}-1} t}{1-c_{2}}\right)-1}}
\end{aligned}
$$


This solution, according to the range of $c_{2}$ and the real or imaginary character of $m_{2}$ is of either neutral or euclidean signature.

In all these four cases the Ricci and Kretsmann scalar are constants and given by $R=-\frac{2 c_{2}}{\left(c_{2}-1\right) \mathrm{ca}^{2}}, k r=\frac{4\left(c_{2}^{2}-2 c_{2}+2\right)}{\left(c_{2}-1\right)^{2} \mathrm{ca}^{4}}$ respenctively. This means that both $\left(c_{2}, c a\right)$ are essential since the determinant of the Wronskian matrix $\frac{\partial(R k r)}{\partial\left(c_{2} c a\right)}$ is not vanishing for all $c a, c_{2} \neq 2$. Moreover the covariant derivative of the Riemann tensor vanishes indicating that there are not higher derivative curvature scalars. We thus have the interesting case of a $C S I$ (constant scalar invariant) space [54]. For the particular value $c_{2}=2$ the geometry is maximally symmetric. If $m_{1} \neq 0$ all branches appearing lead to no solutions.

The reduced Lagrangian density is

$$
\begin{aligned}
\mathcal{L}_{I I I}= & \frac{1}{b^{5} M^{7}}\left(-c_{4} \lambda_{3}^{4} a^{2} M^{6} b^{\prime 2}\right. \\
& -2\left(c_{1}+c_{3}\right) \lambda_{3}^{2} a^{2} b^{2} M^{6} b^{\prime 2}+a^{2} b^{4} M^{4}\left(\lambda_{3}^{2} M^{4} \lambda\right. \\
& \left.+\left(c_{1}+c_{2}+c_{3}\right) u_{0}^{2} b^{\prime 2}\right) \\
& +2 c_{4} \lambda_{3}^{2} a^{2} b^{3} M^{3} u_{0} b^{\prime}\left(u_{0} M^{\prime}-M u_{0}^{\prime}\right) \\
& +b^{6}\left(M^{8}\left(-2+a^{2} \lambda\right)-4 a M^{5} a^{\prime} M^{\prime}\right. \\
& -c_{4} a^{2} u_{0}^{4} M^{\prime 2}+2 c_{4} a^{2} M u_{0}^{3} M^{\prime} u_{0}^{\prime} \\
& -2 a M^{3} u_{0} M^{\prime}\left(2 c_{2} u_{0} a^{\prime}+\left(c_{1}+c_{2}+c_{3}\right) a u_{0}^{\prime}\right) \\
& +a^{2} M^{2} u_{0}^{2}\left(\left(c_{1}+c_{2}+c_{3}\right) M^{\prime 2}-c_{4} u_{0}^{\prime 2}\right) \\
& +M^{4}\left(2\left(c_{1}+2 c_{2}+c_{3}\right) u_{0}^{2} a^{\prime 2}+4 c_{2} a u_{0} a^{\prime} u_{0}^{\prime}\right. \\
& \left.+\left(c_{1}+c_{2}+c_{3}\right) a^{2} u_{0}^{\prime 2}\right) \\
& \left.+M^{6}\left(-a^{2} u_{0}^{2} \lambda+2 a^{\prime 2}+4 a a^{\prime \prime}\right)\right)+ \\
& +2 a b^{5} M^{3}\left(-a M^{2} b^{\prime} M^{\prime}-c_{2} a u_{0}^{2} b^{\prime} M^{\prime}\right. \\
& \left.\left.+c_{2} M u_{0} b^{\prime}\left(2 u_{0} a^{\prime}+a u_{0}^{\prime}\right)+M^{3}\left(2 a^{\prime} b^{\prime}+a b^{\prime \prime}\right)\right)\right)
\end{aligned}
$$

The above Lagrangian density can be seen to be valid since the obtained Euler-Lagrange equations are satisfied by the above given solutions to the reduced equations .

\section{Conclusions}

In the present work we have investigated the dynamical equations of the Einstein-æther theory for the case of an FLRW and an LRS Bianchi type II I geometry. The existence or non-existence of solutions to the reduced equations depends upon the value of various combinations of the initial parameters $c_{I}, I=1 . .4$ entering the action integral (2.1):

In the case of $F L R W$ there exist solutions for any value of the parameters, with the Minkowski metric recovered for the two particular cases $k=0$ and $c_{1}+3 c_{2}+c_{3}=0$. The case $-2+c_{1}+3 c_{2}+c_{3}=0$ is also noteworthy since, on the one hand the dynamics forces $k=0$, while on the other hand $a(t)$ is left free although the time has been fixed by the choice $M(t)=a(t)$. As for a Lagrangian description $\mathcal{L}_{F L R W}$ correctly reproduces the reduced dynamics in all cases, even in that where $a(t)$ is left unspecified. It is noteworthy that the combination of the initial parameters $-c_{1}+3 c_{2}+c_{3}$ entering in (3.7), (3.8), and thus classifying the subsequent solutions found, is exactly what is expected from the findings in [19] applied to the case of an aether field having vanising shear, acceleration and twist (due to the spatial slice being homogeneos and isotropic). However, note that this combination is not the only one that appears in expressions involving the Lagrange multiplier $\lambda(t)$ like (2.4) (when of course the particular ansatz of Sect. 3 is substituted), or (3.3), (3.16); in fact such different combinations are expected so that they cancel out the similar ones of (3.3). The reason for this occurrence is that in [19] the unimodularity of $u^{a}$ is not enforced through a Lagrange multiplier at the level of the action, but rather directly on the dynamical equations.

In the case of LRS Bianchi type II I the situation drastically changes as far as the existense of solutions to the reduced equations is concerned; there are considerably large portions of the parameter space for which no solution exist. For the cases where solutions do exist, the reduced Lagrangian density $\mathcal{L}_{I I I}$ correctly recovers them, indicating that it is valid. It is noteworthy that, when there are no solutions to the reduced equations, the Lagrangian dynamics given by $\mathcal{L}_{I I I}$ also leads to no solutions; thus, for each of these cases, the corresponding Lagrangian constitutes a highly non-trivial example of non-compatible dynamics.

If one would like to make a contact with the Horava gravity, where a physical time $T$ is of main importance, one can say that the high degree of symmetry of the FLRW lineelement constrains the Einstein-æther one-form $u_{a}$ to be a function of the time coordinate only $T \equiv T(t)$. In the case of the LRS Bianchi type I I I geometry, the lesser symmetry permits an initial physical time $T(t, z) \equiv \int u_{0}(t) d t+\lambda_{3} z$; this shows more resemblance to the Horava gravity.

The pure Einstein-Hilbert solutions are not covered by our method because of the use of the uni-modularity equation for the connection between $u_{a}(t)$ and the lapse $M(t)$. However, since the Lagrangian densities $\mathcal{L}_{F L R W}, \mathcal{L}_{I I I}$ are valid for all cases in which solutions to the reduced equations exist, we can easily recover the pure Einstein-Hilbert solutions by just considering the case $T(t)=\mu_{1}$ for the first and $u_{0}(t)=0, \lambda_{3}=0$ for the second.

For the future we intend to investigate more Bianchi models and also add extra matter content e.g. a perfect fluid source in the FLRW. It would be interesting to see which of the properties encountered here persist in these more general cases. 
Acknowledgements AP acknowledges financial support of FONDECYT Grant no. 3160121.

Data Availability Statement This manuscript has no associated data or the data will not be deposited. [Authors' comment: We have not used any data.]

Open Access This article is distributed under the terms of the Creative Commons Attribution 4.0 International License (http://creativecomm ons.org/licenses/by/4.0/), which permits unrestricted use, distribution, and reproduction in any medium, provided you give appropriate credit to the original author(s) and the source, provide a link to the Creative Commons license, and indicate if changes were made. Funded by SCOAP ${ }^{3}$.

\section{References}

1. T. Clifton, P.G. Ferreira, A. Padilla, C. Skordis, Phys. Rep. 513, 1 (2012)

2. L. Amendola, S. Tsujikawa, Dark Energy: Theory and Observations (Cambridge University Press, Cambridge, 2010)

3. S. Perlmutter et al., Astrophys. J. 517, 565 (1998)

4. A.G. Riess et al., Astron. J. 116, 1009 (1998)

5. P. Astier et al., Astrophys. J. 659, 98 (2007)

6. N. Suzuki et al., Astrophys. J. 746, 85 (2012)

7. E. Komatsu et al., Astrophys. J. Suppl. 192, 18 (2011)

8. P.A.R. Ade et al., A\&A 571, A16 (2014)

9. S. Tsujikawa, Lect. Notes Phys. 800, 99 (2010)

10. T. Jacobson, D. Mattingly, Phys. Rev. D 64, 024028 (2001)

11. W. Donnelly, T. Jacobson, Phys. Rev. D 82, 064032 (2010)

12. W. Donnelly, T. Jacobson, Phys. Rev. D 82, 081501 (2010)

13. I. Carruthers, T. Jacobson, Phys. Rev. D 83, 024034 (2011)

14. S.M. Carroll, E.A. Lim, Phys. Rev. D 70, 123525 (2004)

15. C. Heinicke, P. Baekler, F.W. Hehl, Phys. Rev. D 72, 025012 (2005)

16. X. Meng, X. Du, Phys. Lett. B 710, 493 (2012)

17. J.D. Barrow, Phys. Rev. D 85, 047503 (2012)

18. D. Garfinkle, T. Jacobson, Phys. Rev. Lett. 107, 191102 (2011)

19. T. Jacobson, Phys. Rev. D 89, 081501 (2014)

20. P. Sandin, B. Alhulaimi, A. Coley, Phys. Rev. D 87, 044031 (2013)

21. A.A. Coley, G. Leon, P. Sandin, J. Latta, JCAP 12, 010 (2015)

22. J. Latta, G. Leon, A. Paliathanasis, JCAP 16, 051 (2016)

23. A.B. Balakin, V.A. Popov, JCAP 04, 025 (2017)

24. A.B. Balakin, J.P.S. Lemos, Ann. Phys. 350, 454 (2014)

25. C. Eling, T. Jacobson, Class. Quantum Grav. 23, 5625 (2006)
26. C. Eling, T. Jacobson, Class. Quantum Grav. 23, 5643 (2006)

27. A.R. Solomon, J.D. Barrow, Phys. Rev. D 89, 024001 (2014)

28. E. Barausse, T. Jacobson, T.P. Sotiriou, Phys. Rev. D 83, 124043 (2011)

29. E. Barausse, T.P. Sotiriou, I. Vega, Phys. Rev. D 93, 044044 (2016)

30. C. Gao, Y.-G. Sen, Phys. Rev. D 88, 103508 (2013)

31. B. Alhulaimi, A. Coley, P. Sandin, J. Math. Phys. 54, 042503 (2013)

32. G. Morandi, G. Ferrario, G. LoVecchio, G. Marmo, C. Rubano, Phys. Rep. 188, 147 (1990)

33. M.A.H. MacCallum, H. Taub, Commun. Math. Phys. 25, 173-189 (1972)

34. T. Christodoulakis, E. Korfiatis, Nuov Cim B 109, 1155 (1994). https://doi.org/10.1007/BF02726679

35. T. Christodoulakis, E. Korfiatis, A. Paschos, Phys. Rev. D 54, 2691 2698 (1996) [Erratum-ibid. D 56, 5279-5280 (1997)]

36. A. Maciejewski, M. Szydlowski, J. Phys. A Math. Gen. 31, 2031 (1998)

37. A. Ferragut, J. Llibre, C. Pantazi, Chaos Solitons Fract. 48, 12 (2013)

38. A. Ferragut, J. Llibre, C. Pantazi, Chaos 23, 013119 (2013)

39. J. Llibre, J. Math. Phys. 46, 072901 (2015)

40. P.G.L. Leach, M.C. Nucci, S. Cotsakis, J. Nonlinear Math. Phys. 8, 475 (2001)

41. S. Basilakos, M. Tsamparlis, A. Paliathanasis, Phys. Rev. D 83, 103512 (2011)

42. N. Dimakis, A. Giacomini, A. Paliathanasis, EPJC 77, 458 (2017)

43. K.V. Kuchar, M.P. Jr, Ryan. Phys. Rev. D 40, 3982 (1989)

44. J. Martin, Phys. Rev. D 49, 5086 (1994)

45. C. Simeone, J. Math. Phys. 39, 3131 (1998)

46. T. Christodoulakis, N. Dimakis, P.A. Terzis, G. Doulis, Phys. Rev. D 90, 024052 (2014)

47. T. Christodoulakis, N. Dimakis, P.A. Terzis, B. Vakili, E. Melas, Th Grammenos, Phys. Rev. D 89, 044031 (2014)

48. T. Christodoulakis, N. Dimakis, P.A. Terzis, G. Doulis, Th Grammenos, J. Geom. Phys. 71, 127 (2013)

49. A. Paliathanasis, M. Tsamparlis, S. Basilakos, J.D. Barrow, Phys. Rev. D 93, 043528 (2016)

50. C.W. Misner, Phys. Rev. Lett. 22, 1071 (1969)

51. M.P.J. Rayan, L.C. Shepley, Homogeneous Relativistic Cosmologies (Princeton University Press, Princeton, 1975)

52. C.W. Misner, Phys. Rev. D 186, 1319 (1969)

53. M.A.H. MacCallumn, in General Relativity: An Einstein Centenary Survey, ed. by W.S.W. Hawking, W. Israel (Cambridge University Press, Cambridge, 1979)

54. A. Coley, S. Hervik, N. Pelavas, Class. Quant. Grav. 23, 3053 (2006) 